\title{
Hermite-based unified Apostol-Bernoulli, Euler and Genocchi polynomials
}

\section{Mehmet Ali Özarslan*}

\section{"Correspondence:}

mehmetali.ozarslan@emu.edu.tr Department of Mathematics,

Faculty of Arts of Sciences, Eastern

Mediterranean University,

Gazimagusa, North Cyprus,

Mersin 10, Turkey

\begin{abstract}
In this paper, we introduce a unified family of Hermite-based Apostol-Bernoulli, Euler and Genocchi polynomials. We obtain some symmetry identities between these polynomials and the generalized sum of integer powers. We give explicit closed-form formulae for this unified family. Furthermore, we prove a finite series relation between this unification and $3 d$-Hermite polynomials.

MSC: Primary 11B68; secondary 33C05

Keywords: Hermite-based Apostol-Bernoulli polynomials; Hermite-based Apostol-Euler polynomials; Hermite-based Apostol-Genocchi polynomials; generalized sum of integer powers; generalized sum of alternative integer powers
\end{abstract}

\section{Introduction}

Recently, Khan et al. [1] introduced the Hermite-based Appell polynomials via the generating function

$$
\mathcal{G}(x, y, z ; t)=A(t) \exp (\mathcal{M} t)
$$

where

$$
\mathcal{M}=x+2 y \frac{\partial}{\partial x}+3 z \frac{\partial^{2}}{\partial x^{2}}
$$

is the multiplicative operator of the 3-variable Hermite polynomials, which are defined by

$$
\exp \left(x t+y t^{2}+z t^{3}\right)=\sum_{n=0}^{\infty} H_{n}^{(3)}(x, y, z) \frac{t^{n}}{n !}
$$

and

$$
A(t)=\sum_{n=0}^{\infty} a_{n} t^{n}, \quad a_{0} \neq 0 .
$$

By using the Berry decoupling identity,

$$
e^{A+B}=e^{m^{2} / 12} e^{\left(\left(\frac{-m}{2}\right) A^{1 / 2}+A\right)} e^{B}, \quad[A, B]=m A^{1 / 2}
$$

(C) 2013 Özarslan; licensee Springer. This is an Open Access article distributed under the terms of the Creative Commons Attribution License (http://creativecommons.org/licenses/by/2.0), which permits unrestricted use, distribution, and reproduction in any medium, provided the original work is properly cited. 
they obtained the generating function of the Hermite-based Appell polynomials ${ }_{H} A_{n}(x$, $y, z)$ as

$$
\mathcal{G}(x, y, z ; t)=A(t) \exp \left(x t+y t^{2}+z t^{3}\right)=\sum_{n=0}^{\infty}{ }_{H} A_{n}(x, y, z) \frac{t^{n}}{n !}
$$

Letting $A(t)=\frac{t}{e^{t}-1}$, they defined Hermite-Bernoulli polynomials ${ }_{H} B_{n}(x, y, z)$ by

$$
\frac{t}{e^{t}-1} \exp \left(x t+y t^{2}+z t^{3}\right)=\sum_{n=0}^{\infty} H_{H} B_{n}(x, y, z) \frac{t^{n}}{n !}, \quad|t|<2 \pi
$$

For $A(t)=\frac{2}{e^{t}+1}$, they defined Hermite-Euler polynomials ${ }_{H} E_{n}(x, y, z)$ by

$$
\frac{2}{e^{t}+1} \exp \left(x t+y t^{2}+z t^{3}\right)=\sum_{n=0}^{\infty}{ }_{H} E_{n}(x, y, z) \frac{t^{n}}{n !}, \quad|t|<\pi
$$

and for $A(t)=\frac{2 t}{e^{t}+1}$, they defined Hermite-Genocchi polynomials ${ }_{H} G_{n}(x, y, z)$ by

$$
\frac{2 t}{e^{t}+1} \exp \left(x t+y t^{2}+z t^{3}\right)=\sum_{n=0}^{\infty}{ }_{H} G_{n}(x, y, z) \frac{t^{n}}{n !}, \quad|t|<\pi .
$$

Recently, the author considered the following unification of the Apostol-Bernoulli, Euler and Genocchi polynomials

$$
\begin{gathered}
f_{a, b}^{(\alpha)}(x ; t ; k, \beta):=\left(\frac{2^{1-k} t^{k}}{\beta^{b} e^{t}-a^{b}}\right)^{\alpha} e^{x t}=\sum_{n=0}^{\infty} P_{n, \beta}^{(\alpha)}(x ; k, a, b) \frac{t^{n}}{n !} \\
\left(k \in \mathbb{N}_{0} ; a, b \in \mathbb{R} \backslash\{0\} ; \alpha, \beta \in \mathbb{C}\right)
\end{gathered}
$$

and obtained the explicit representation of this unified family, in terms of Gaussian hypergeometric function. Some symmetry identities and multiplication formula are also given in [2]. Note that the family of polynomials $P_{n, \beta}^{(1)}(x, y, z ; k, a, b)$ was investigated in [3].

We organize the paper as follows.

In Section 2, we introduce the unification of the Hermite-based generalized ApostolBernoulli, Euler and Genocchi polynomials ${ }_{H} P_{n, \beta}^{(\alpha)}(x, y, z ; k, a, b)$ and give summation formulas for this unification. In Section 3, we obtain some symmetry identities for these polynomials. In Section 4, we give explicit closed-form formulae for this unified family. Furthermore, we prove a finite series relation between this unification and $3 d$-Hermite polynomials.

\section{Hermite-based generalized Apostol-Bernoulli, Euler and Genocchi polynomials}

In this paper, we consider the following general class of polynomials:

$$
\begin{aligned}
& f_{a, b}^{(\alpha)}(x, y, z ; t ; k, \beta):=\left(\frac{2^{1-k} t^{k}}{\beta^{b} e^{t}-a^{b}}\right)^{\alpha} e^{x t+y t^{2}+z t^{3}}=\sum_{n=0}^{\infty}{ }_{H} P_{n, \beta}^{(\alpha)}(x, y, z ; k, a, b) \frac{t^{n}}{n !} \\
& \left(k \in \mathbb{N}_{0} ; a, b \in \mathbb{R} \backslash\{0\} ; \alpha, \beta \in \mathbb{C}\right) .
\end{aligned}
$$


For the existence of the expansion, we need

(i) $|t|<2 \pi$ when $\alpha \in \mathbb{C}, k=1$ and $\left(\frac{\beta}{a}\right)^{b}=1 ;|t|<2 \pi$ when $\alpha \in \mathbb{N}_{0}, k=2,3, \ldots$ and $\left(\frac{\beta}{a}\right)^{b}=1 ;|t|<\left|b \log \left(\frac{\beta}{a}\right)\right|$ when $\alpha \in \mathbb{N}_{0}, k \in \mathbb{N}$ and $\left(\frac{\beta}{a}\right)^{b} \neq 1$ (or $\neq-1$ ); $x, y, z \in \mathbb{R}, \beta \in \mathbb{C}$, $a, b \in \mathbb{C} /\{0\} ; 1^{\alpha}:=1$;

(ii) $|t|<\pi$ when $\left(\frac{\beta}{a}\right)^{b}=-1 ;|t|<\left|b \log \left(\frac{\beta}{a}\right)\right|$ when $\left(\frac{\beta}{a}\right)^{b} \neq-1 ; x, y, z \in \mathbb{R}, k=0, \alpha, \beta \in \mathbb{C}$, $a, b \in \mathbb{C} /\{0\} ; 1^{\alpha}:=1$;

(iii) $|t|<\pi$ when $\alpha \in \mathbb{N}_{0}$ and $\left(\frac{\beta}{a}\right)^{b}=-1 ; x, y, z \in \mathbb{R}, k \in \mathbb{N}, \beta \in \mathbb{C}, a, b \in \mathbb{C} /\{0\} ; 1^{\alpha}:=1$, where $w=|w| e^{i \theta},-\pi \leq \theta<\pi$ and $\log (w)=\log (|w|)+i \theta$.

For $k=a=b=1$ and $\beta=\lambda$ in (2.1), we define the following.

Definition 2.1 Let $\alpha \in \mathbb{N}_{0}, \lambda$ be an arbitrary (real or complex) parameter and $x, y, z \in \mathbb{R}$. The Hermite-based generalized Apostol-Bernoulli polynomials are defined by

$$
\begin{aligned}
& \left(\frac{t}{\lambda e^{t}-1}\right)^{\alpha} \exp \left(x t+y t^{2}+z t^{3}\right)=\sum_{n=0}^{\infty}{ }_{H} \mathcal{B}_{n}^{(\alpha)}(x, y, z ; \lambda) \frac{t^{n}}{n !} \\
& (|t|<2 \pi \text { when } \alpha \in \mathbb{C} \text { and } \lambda=1 ;|t|<|\log (\lambda)| \\
& \text { when } \left.\alpha \in \mathbb{N}_{0} \text { and } \lambda \neq 1 ; x, y, z \in \mathbb{R} ; 1^{\alpha}:=1\right) .
\end{aligned}
$$

It is clear that

$$
{ }_{H} P_{n, \lambda}^{(\alpha)}(x, y, z ; 1,1,1)={ }_{H} \mathcal{B}_{n}^{(\alpha)}(x, y, z ; \lambda) .
$$

Some special cases of the Hermite-based generalized Apostol-Bernoulli polynomials (some of which are definition) are listed below:

- ${ }_{H} \mathcal{B}_{n}^{(1)}(x, y, z ; \lambda):={ }_{H} \mathcal{B}_{n}(x, y, z ; \lambda)$ is called Hermite-based Apostol-Bernoulli polynomials.

- ${ }_{H} \mathcal{B}_{n}(x, y, z ; 1)={ }_{H} B_{n}(x, y, z)$ is the Hermite-Bernoulli polynomials.

- ${ }_{H} \mathcal{B}_{n}(x, 0,0 ; \lambda):=\mathcal{B}_{n}(x ; \lambda)$ is the Apostol-Bernoulli polynomials (see [4-7]). When $\lambda=1$, we have the classical Bernoulli polynomials.

- $\mathcal{B}_{n}(0 ; \lambda):=\mathcal{B}_{n}(\lambda)$ are the Apostol-Bernoulli numbers. $\lambda=1$ gives the classical Bernoulli numbers.

Setting $k+1=-a=b=1$ and $\beta=\lambda$ in (2.1), we get the following.

Definition 2.2 Let $\alpha$ and $\lambda(\neq-1)$ be an arbitrary (real or complex) parameter and $x, y, z \in \mathbb{R}$. The Hermite-based generalized Apostol-Euler polynomials are defined by

$$
\begin{aligned}
& \left(\frac{2}{\lambda e^{t}+1}\right)^{\alpha} \exp \left(x t+y t^{2}+z t^{3}\right)=\sum_{n=0}^{\infty}{ }_{H} \mathcal{E}_{n}^{(\alpha)}(x, y, z ; \lambda) \frac{t^{n}}{n !} \\
& \left(|t|<\pi \text { when } \lambda=1 ;|t|<|\log (-\lambda)| \text { when } \lambda \neq 1 ; x, y, z \in \mathbb{R}, \alpha \in \mathbb{C} ; 1^{\alpha}:=1\right) .
\end{aligned}
$$

Obviously, we have

$$
{ }_{H} P_{n, \lambda}^{(\alpha)}(x, y, z ; 0,-1,1)={ }_{H} \mathcal{E}_{n}^{(\alpha)}(x, y, z ; \lambda) .
$$

Some special cases of the Hermite-based generalized Apostol-Euler polynomials (some of which are definition) are listed below: 
- ${ }_{H} \mathcal{E}_{n}^{(1)}(x, y, z ; \lambda):={ }_{H} \mathcal{E}_{n}(x, y, z ; \lambda)$ is called Hermite-based Apostol-Euler polynomials.

- ${ }_{H} \mathcal{E}_{n}(x, y, z ; 1)={ }_{H} E_{n}(x, y, z)$ is the Hermite-Euler polynomials.

- ${ }_{H} \mathcal{E}_{n}(x, 0,0 ; \lambda):=\mathcal{E}_{n}(x ; \lambda)$ is the Apostol-Euler polynomials (see [8]). For $\lambda=1$, we have the classical Euler polynomials.

- $2^{n} \mathcal{E}_{n}\left(\frac{1}{2} ; \lambda\right):=\mathcal{E}_{n}(\lambda)$ are the Apostol-Euler numbers. The case $\lambda=1$ gives the classical Euler numbers.

Choosing $k=-2 a=b=1$ and $2 \beta=\lambda$ in (2.1), we define the following.

Definition 2.3 Let $\alpha$ and $\lambda(\neq-1)$ be an arbitrary (real or complex) parameter and $x, y, z \in \mathbb{R}$. The Hermite-based generalized Apostol-Genocchi polynomials are defined by

$$
\begin{aligned}
\left(\frac{2 t}{\lambda e^{t}+1}\right)^{\alpha} \exp \left(x t+y t^{2}+z t^{3}\right) & =\sum_{n=0}^{\infty}{ }_{H} \mathcal{G}_{n}^{(\alpha)}(x, y, z ; \lambda) \frac{t^{n}}{n !} \\
\left(|t|<\pi \text { when } \alpha \in \mathbb{N}_{0} \text { and } \lambda\right. & =1 ;|t|<|\log (-\lambda)|
\end{aligned}
$$

when $\alpha \in \mathbb{N}_{0}$ and $\left.\lambda \neq 1 ; x, y, z \in \mathbb{R} ; 1^{\alpha}:=1\right)$.

It is easily seen that

$$
{ }_{H} P_{n, \frac{\lambda}{2}}^{(\alpha)}\left(x, y, z ; 1, \frac{-1}{2}, 1\right)={ }_{H} \mathcal{G}_{n}^{\alpha}(x, y, z ; \lambda)
$$

Some special cases of the Hermite-based generalized Apostol-Genocchi polynomials (some of which are definition) are listed below:

- ${ }_{H} \mathcal{G}_{n}^{(1)}(x, y, z ; \lambda):={ }_{H} \mathcal{G}_{n}(x, y, z ; \lambda)$ is called Hermite-based Apostol-Genocchi polynomials.

- ${ }_{H} \mathcal{G}_{n}(x, y, z ; 1)={ }_{H} G_{n}(x, y, z)$ is the Hermite-Genocchi polynomials.

- ${ }_{H} \mathcal{G}_{n}(x, 0,0 ; \lambda):=\mathcal{G}_{n}(x ; \lambda)$ is the Apostol-Genocchi polynomials (see $\left.[9,10]\right)$. When $\lambda=1$, we have the classical Genocchi polynomials.

- $\mathcal{G}_{n}(0 ; \lambda):=\mathcal{G}_{n}(\lambda)$ are the Apostol-Genocchi numbers. $\lambda=1$ gives the classical Genocchi numbers.

Finally we define the unified Hermite-based Apostol polynomials by

$$
\begin{aligned}
& f_{a, b}^{(1)}(x ; t ; k, \beta):=\frac{2^{1-k} t^{k}}{\beta^{b} e^{t}-a^{b}} e^{x t+y t^{2}+z t^{3}}=\sum_{n=0}^{\infty}{ }_{H} P_{n, \beta}(x, y, z ; k, a, b) \frac{t^{n}}{n !} \\
& \left(k \in \mathbb{N}_{0} ; a, b \in \mathbb{R} \backslash\{0\} ; \beta \in \mathbb{C}\right) .
\end{aligned}
$$

Thus it is clear that ${ }_{H} P_{n, \beta}(x, y, z ; k, a, b)={ }_{H} P_{n, \beta}^{(1)}(x, y, z ; k, a, b)$ and that we have the following observations at once:

- ${ }_{H} P_{n, \lambda}(x, y, z ; 1,1,1)={ }_{H} \mathcal{B}_{n}(x, y, z ; \lambda)$ are the Hermite-based Apostol-Bernoulli polynomials.

- ${ }_{H} P_{n, \lambda}(x, y, z ; 0,-1,1)={ }_{H} \mathcal{E}(x, y, z ; \lambda)$ are the Hermite-based Apostol-Euler polynomials.

- ${ }_{H} P_{n, \frac{\lambda}{2}}\left(x, y, z ; 1, \frac{-1}{2}, 1\right)={ }_{H} \mathcal{G}_{n}(x, y, z ; \lambda)$ are the Hermite-based Apostol-Genocchi polynomials. 
For the other generalization, we refer [11-25] and [26]. Now we give some relations between the above mentioned Apostol polynomials.

Using (2.1), we get the following identity at once.

Theorem 2.1 Let $\alpha, k \in \mathbb{N}_{0} ; a, b \in \mathbb{R} \backslash\{0\} ; \beta \in \mathbb{C}$ be such that the conditions (i)-(iii) are satisfied. Then, the following relation

$$
\sum_{r=0}^{n}\left(\begin{array}{l}
n \\
r
\end{array}\right){ }_{H} P_{n-r, \beta}^{(\alpha)}(x, y, z ; k, a, b){ }_{H} P_{r, \beta}^{(\alpha)}(u, v, w ; k, a, b)={ }_{H} P_{n, \beta}^{(\alpha)}(x+u, y+v, z+w ; k, a, b)
$$

holds true.

Corollary 2.2 For each $n \in \mathbb{N}$, the following relation

$$
\sum_{k=0}^{n}\left(\begin{array}{l}
n \\
k
\end{array}\right){ }_{H} \mathcal{B}_{n-k}^{(\alpha)}(x, y, z ; \lambda){ }_{H} \mathcal{B}_{k}^{(\beta)}(u, v, w ; \lambda)={ }_{H} \mathcal{B}_{n}^{(\alpha+\beta)}(x+u, y+v, z+w ; \lambda)
$$

holds true for the Hermite-based generalized Apostol-Bernoulli polynomials.

Corollary 2.3 For each $n \in \mathbb{N}$, the following relation

$$
\sum_{k=0}^{n}\left(\begin{array}{l}
n \\
k
\end{array}\right){ }_{H} \mathcal{E}_{n-k}^{(\alpha)}(x, y, z ; \lambda)_{H} \mathcal{E}_{k}^{(\beta)}(u, v, w ; \lambda)={ }_{H} \mathcal{E}_{n}^{(\alpha+\beta)}(x+u, y+v, z+w ; \lambda)
$$

holds true for the Hermite-based generalized Apostol-Euler polynomials.

Corollary 2.4 For each $n \in \mathbb{N}$, the following relation

$$
\sum_{k=0}^{n}\left(\begin{array}{l}
n \\
k
\end{array}\right){ }_{H} \mathcal{G}_{n-k}^{(\alpha)}(x, y, z ; \lambda)_{H} \mathcal{G}_{k}^{(\beta)}(u, v, w ; \lambda)={ }_{H} \mathcal{G}_{n}^{(\alpha+\beta)}(x+u, y+v, z+w ; \lambda)
$$

holds true for the Hermite-based generalized Apostol-Genocchi polynomials.

Theorem 2.5 For each $n \in \mathbb{N}$, the following relation

$$
\sum_{k=0}^{n}\left(\begin{array}{l}
n \\
k
\end{array}\right){ }_{H} \mathcal{B}_{n-k}^{(\alpha)}(x, y, z ; \lambda)_{H} \mathcal{E}_{k}^{(\alpha)}(u, v, w ; \lambda)=2_{H}^{n} \mathcal{B}_{n}^{(\alpha)}\left(\frac{x+u}{2}, \frac{y+v}{4}, \frac{z+w}{8} ; \lambda^{2}\right)
$$

holds true between the Hermite-based generalized Apostol-Bernoulli and Euler polynomials.

Proof By direct calculations, we have

$$
\begin{aligned}
& \sum_{n=0}^{\infty}{ }_{H} \mathcal{B}_{n}^{(\alpha)}\left(\frac{x+u}{2}, \frac{y+v}{4}, \frac{z+w}{8} ; \lambda^{2}\right) \frac{(2 t)^{n}}{n !} \\
& =\left(\frac{2 t}{\lambda^{2} e^{2 t}-1}\right)^{\alpha} \exp \left[\left(\frac{x+u}{2}\right) 2 t+\left(\frac{y+v}{4}\right)(2 t)^{2}+\left(\frac{z+w}{8}\right)(2 t)^{3}\right] \\
& =\left(\frac{t}{\lambda e^{t}-1}\right)^{\alpha} \exp \left(x t+y t^{2}+z t^{3}\right)\left(\frac{2}{\lambda e^{t}+1}\right)^{\alpha} \exp \left(u t+v t^{2}+w t^{3}\right)
\end{aligned}
$$




$$
\begin{aligned}
& =\sum_{n=0}^{\infty}{ }_{H} \mathcal{B}_{n}^{(\alpha)}(x, y, z ; \lambda) \frac{t^{n}}{n !} \sum_{k=0}^{\infty}{ }_{H} \mathcal{E}_{k}^{(\alpha)}(u, v, w ; \lambda) \frac{t^{k}}{k !} \\
& =\sum_{n=0}^{\infty} \sum_{k=0}^{n}\left(\begin{array}{l}
n \\
k
\end{array}\right){ }_{H} \mathcal{B}_{n-k}^{(\alpha)}(x, y, z ; \lambda)_{H} \mathcal{E}_{k}^{(\alpha)}(u, v, w ; \lambda) \frac{t^{n}}{n !} .
\end{aligned}
$$

Comparing the coefficients of $\frac{t^{n}}{n !}$ on both sides, we get the result.

\section{Symmetry identities for the unified family}

For each $k \in \mathbb{N}_{0}$, the sum $S_{k}(n)=\sum_{i=0}^{n} i^{k}$ is known as the power sum and we have the following generating relation:

$$
\sum_{k=0}^{\infty} S_{k}(n) \frac{t^{k}}{k !}=1+e^{t}+e^{2 t}+\cdots+e^{n t}=\frac{e^{(n+1) t}-1}{e^{t}-1}
$$

For an arbitrary real or complex $\lambda$, the generalized sum of integer powers $S_{k}(n, \lambda)$ is defined, in [27], via the following generating relation:

$$
\sum_{k=0}^{\infty} S_{k}(n, \lambda) \frac{t^{k}}{k !}=\frac{\lambda e^{(n+1) t}-1}{\lambda e^{t}-1}
$$

It clear that $S_{k}(n, 1)=S_{k}(n)$.

For each $k \in \mathbb{N}_{0}$, the sum $M_{k}(n)=\sum_{i=0}^{n}(-1)^{k} i^{k}$ is known as the sum of alternative integer powers. The following generating relation is straightforward:

$$
\sum_{k=0}^{\infty} M_{k}(n) \frac{t^{k}}{k !}=1-e^{t}+e^{2 t}-\cdots+(-1)^{n} e^{n t}=\frac{1-\left(-e^{t}\right)^{(n+1)}}{e^{t}+1} .
$$

For an arbitrary real or complex $\lambda$, the generalized sum of alternative integer powers $M_{k}(n, \lambda)$ is defined, in [27], by

$$
\sum_{k=0}^{\infty} M_{k}(n, \lambda) \frac{t^{k}}{k !}=\frac{1-\lambda\left(-e^{t}\right)^{(n+1)}}{\lambda e^{t}+1} .
$$

Clearly $M_{k}(n, 1)=M_{k}(n)$. On the other hand, if $n$ is even, then

$$
S_{k}(n,-\lambda)=M_{k}(n, \lambda)
$$

We start by obtaining certain symmetry identities, which includes the results given in [28-32] and [27], when $y=z=0$.

Theorem 3.1 Let c, $d, m \in \mathbb{N}, n \in \mathbb{N}_{0}$ be such that the conditions (i)-(iii) are satisfied with treplaced by ct and $d t$. Then we have the following symmetry identity:

$$
\begin{aligned}
& \sum_{r=0}^{n}\left(\begin{array}{l}
n \\
r
\end{array}\right) c^{n-r} d^{r+k}{ }_{H} P_{n-r, \beta}^{(m)}\left(d x, d^{2} y, d^{3} z ; k, a, b\right) \\
& \quad \times \sum_{l=0}^{r}\left(\begin{array}{l}
r \\
l
\end{array}\right) S_{l}\left(c-1 ;\left(\frac{\beta}{a}\right)^{b}\right){ }_{H} P_{r-l, \beta}^{(m-1)}\left(c X, c^{2} Y, c^{3} Z ; k, a, b\right)
\end{aligned}
$$




$$
\begin{aligned}
= & \sum_{r=0}^{n}\left(\begin{array}{l}
n \\
r
\end{array}\right) d^{n-r} c^{r+k}{ }_{H} P_{n-r, \beta}^{(m)}\left(c x, c^{2} y, c^{3} z ; k, a, b\right) \\
& \times \sum_{l=0}^{r}\left(\begin{array}{l}
r \\
l
\end{array}\right) S_{l}\left(d-1 ;\left(\frac{\beta}{a}\right)^{b}\right){ }_{H} P_{r-l, \beta}^{(m-1)}\left(d X, d^{2} Y, d^{3} Z ; k, a, b\right) .
\end{aligned}
$$

Proof Let

$$
G(t):=\frac{2^{(1-k)(2 m-1)} t^{2 k m-k} e^{c d x t+y(c d t)^{2}+z(c d t)^{3}}\left(\beta^{b} e^{c d t}-a^{b}\right) e^{c d X t+Y(c d t)^{2}+Z(c d t)^{3}}}{\left(\beta^{b} e^{c t}-a^{b}\right)^{m}\left(\beta^{b} e^{d t}-a^{b}\right)^{m}} .
$$

Expanding $G(t)$ into a series, we get

$$
\begin{aligned}
G(t)= & \frac{1}{c^{k m} d^{k(m-1)}}\left(\frac{2^{1-k} c^{k} t^{k}}{\beta^{b} e^{c t}-a^{b}}\right)^{m} e^{c d x t+y(c d t)^{2}+z(c d t)^{3}}\left(\frac{\beta^{b} e^{c d t}-a^{b}}{\beta^{b} e^{d t}-a^{b}}\right) \\
& \times\left(\frac{2^{1-k} d^{k} t^{k}}{\beta^{b} e^{d t}-a^{b}}\right)^{m-1} e^{c d X t+Y(c d t)^{2}+Z(c d t)^{3}} \\
= & \frac{1}{c^{k m} d^{k(m-1)}}\left[\sum_{n=0}^{\infty}{ }_{H} P_{n, \beta}^{(m)}\left(d x, d^{2} y, d^{3} z ; k, a, b\right) \frac{(c t)^{n}}{n !}\right]\left[\sum_{l=0}^{\infty} S_{l}\left(c-1 ;\left(\frac{\beta}{a}\right)^{b}\right) \frac{(d t)^{l}}{l !}\right] \\
& \times\left[\sum_{r=0}^{\infty}{ }_{H} P_{r, \beta}^{(m-1)}\left(c X, c^{2} Y, c^{3} Z ; k, a, b\right) \frac{(d t)^{r}}{r !}\right]
\end{aligned}
$$

Now, using Corollary 2 in [33, p.890], we get

$$
\begin{aligned}
G(t)= & \frac{1}{c^{k m} d^{k m}} \sum_{n=0}^{\infty}\left[\sum_{r=0}^{n}\left(\begin{array}{l}
n \\
r
\end{array}\right) c^{n-r} d^{r+k}{ }_{H} P_{n-r, \beta}^{(m)}\left(d x, d^{2} y, d^{3} z ; k, a, b\right)\right. \\
& \left.\times \sum_{l=0}^{r}\left(\begin{array}{l}
r \\
l
\end{array}\right) S_{l}\left(c-1 ;\left(\frac{\beta}{a}\right)^{b}\right){ }_{H} P_{r-l, \beta}^{(m-1)}\left(c X, c^{2} Y, c^{3} Z ; k, a, b\right)\right] \frac{t^{n}}{n !}
\end{aligned}
$$

In a similar manner,

$$
\begin{aligned}
G(t)= & \frac{1}{d^{k m} c^{k(m-1)}}\left(\frac{2^{1-k} d^{k} t^{k}}{\beta^{b} e^{c t}-a^{b}}\right)^{m} e^{c d x t+y(c d t)^{2}+z(c d t)^{3}}\left(\frac{\beta^{b} e^{c d t}-a^{b}}{\beta^{b} e^{d t}-a^{b}}\right) \\
& \times\left(\frac{2^{1-k} c^{k} t^{k}}{\beta^{b} e^{d t}-a^{b}}\right)^{m-1} e^{c d X t+Y(c d t)^{2}+Z(c d t)^{3}} \\
= & \frac{1}{c^{k m} d^{k m}} \sum_{n=0}^{\infty}\left[\sum_{r=0}^{n}\left(\begin{array}{l}
n \\
r
\end{array}\right) d^{n-r} c^{r+k}{ }_{H} P_{n-r, \beta}^{(m)}\left(c x, c^{2} y, c^{3} z ; k, a, b\right)\right. \\
& \left.\times \sum_{l=0}^{r}\left(\begin{array}{l}
r \\
l
\end{array}\right) S_{l}\left(d-1 ;\left(\frac{\beta}{a}\right)^{b}\right){ }_{H} P_{r-l, \beta}^{(m-1)}\left(d X, d^{2} Y, d^{3} Z ; k, a, b\right)\right] \frac{t^{n}}{n !} .
\end{aligned}
$$

From (3.2) and (3.3), we get the result.

For $k=a=b=1$ and $\beta=\lambda$ we get the following corollary at once. 
Corollary 3.2 For all $c, d, m \in \mathbb{N}, n \in \mathbb{N}_{0}, \lambda \in \mathbb{C}$, we have the following symmetry identity for the Hermite based generalized Apostol-Bernoulli polynomials:

$$
\begin{aligned}
& \sum_{r=0}^{n}\left(\begin{array}{l}
n \\
r
\end{array}\right) c^{n-r} d^{r+1}{ }_{H} \mathcal{B}_{n-r}^{(m)}\left(d x, d^{2} y, d^{3} z, \lambda\right) \\
& \quad \times \sum_{l=0}^{r}\left(\begin{array}{l}
r \\
l
\end{array}\right) S_{l}(c-1 ; \lambda)_{H} \mathcal{B}_{r-l}^{(m-1)}\left(c X, c^{2} Y, c^{3} Z, \lambda\right) \\
& =\sum_{r=0}^{n}\left(\begin{array}{l}
n \\
r
\end{array}\right) d^{n-r} c^{r+1}{ }_{H} \mathcal{B}_{n-r}^{(m)}\left(c x, c^{2} y, c^{3} z, \lambda\right) \\
& \quad \times \sum_{l=0}^{r}\left(\begin{array}{l}
r \\
l
\end{array}\right) S_{l}(d-1 ; \lambda)_{H} \mathcal{B}_{r-l}^{(m-1)}\left(d X, d^{2} Y, d^{3} Z, \lambda\right) .
\end{aligned}
$$

For $k+1=-a=b=1$ and $\beta=\lambda$ we get, by considering (3.1) that

Corollary 3.3 For all $m \in \mathbb{N}, n \in \mathbb{N}_{0}, \lambda \in \mathbb{C}$, we have for each pair of positive even integers $c$ and $d$, or for each pair of positive odd integers $c$ and $d$,

$$
\begin{aligned}
& \sum_{r=0}^{n}\left(\begin{array}{l}
n \\
r
\end{array}\right) c^{n-r} d^{r+1}{ }_{H} \mathcal{E}_{n-r}^{(m)}\left(d x, d^{2} y, d^{3} z, \lambda\right) \\
& \quad \times \sum_{l=0}^{r}\left(\begin{array}{l}
r \\
l
\end{array}\right) M_{l}(c-1 ; \lambda)_{H} \mathcal{E}_{r-l}^{(m-1)}\left(c X, c^{2} Y, c^{3} Z, \lambda\right) \\
& =\sum_{r=0}^{n}\left(\begin{array}{l}
n \\
r
\end{array}\right) d^{n-r} c^{r+1}{ }_{H} \mathcal{E}_{n-r}^{(m)}\left(c x, c^{2} y, c^{3} z, \lambda\right) \\
& \quad \times \sum_{l=0}^{r}\left(\begin{array}{l}
r \\
l
\end{array}\right) M_{l}(d-1 ; \lambda)_{H} \mathcal{E}_{r-l}^{(m-1)}\left(d X, d^{2} Y, d^{3} Z, \lambda\right) .
\end{aligned}
$$

Letting $k=-2 a=b=1$ and $2 \beta=\lambda$ and taking into account (3.1) that we have the following.

Corollary 3.4 For all $m \in \mathbb{N}, n \in \mathbb{N}_{0}, \lambda \in \mathbb{C}$, we have for each pair of positive even integers $c$ and $d$, or for each pair of positive odd integers $c$ and $d$, that

$$
\begin{aligned}
& \sum_{r=0}^{n}\left(\begin{array}{l}
n \\
r
\end{array}\right) c^{n-r} d^{r+1}{ }_{H} \mathcal{G}_{n-r}^{(m)}\left(d x, d^{2} y, d^{3} z, \lambda\right) \\
& \quad \times \sum_{l=0}^{r}\left(\begin{array}{l}
r \\
l
\end{array}\right) M_{l}(c-1 ; \lambda)_{H} \mathcal{G}_{r-l}^{(m-1)}\left(c X, c^{2} Y, c^{3} Z, \lambda\right) \\
& =\sum_{r=0}^{n}\left(\begin{array}{l}
n \\
r
\end{array}\right) d^{n-r} c^{r+1}{ }_{H} \mathcal{G}_{n-r}^{(m)}\left(c x, c^{2} y, c^{3} z, \lambda\right) \\
& \quad \times \sum_{l=0}^{r}\left(\begin{array}{l}
r \\
l
\end{array}\right) M_{l}(d-1 ; \lambda)_{H} \mathcal{G}_{r-l}^{(m-1)}\left(d X, d^{2} Y, d^{3} Z, \lambda\right) .
\end{aligned}
$$




\section{Closed-form formulae for Hermite-based generalized Apostol polynomials}

In this section, taking into account the relations

$$
\begin{aligned}
& f_{a, b}^{(\alpha)}(x, y, z ; t ; k, \beta):=\left(\frac{2^{1-k} t^{k}}{\beta^{b} e^{t}-a^{b}}\right)^{\alpha} e^{x t+y t^{2}+z t^{3}}=\sum_{n=0}^{\infty}{ }_{H} P_{n, \beta}^{(\alpha)}(x, y, z ; k, a, b) \frac{t^{n}}{n !}, \\
& f_{a, b}^{(1)}(x, y, z ; t ; k, \beta):=\left(\frac{2^{1-k} t^{k}}{\beta^{b} e^{t}-a^{b}}\right) e^{x t+y t^{2}+z t^{3}}=\sum_{n=0}^{\infty}{ }_{H} P_{n, \beta}(x, y, z ; k, a, b) \frac{t^{n}}{n !},
\end{aligned}
$$

we observe the following fact:

$$
\left[f_{a, b}^{(1)}\left(\frac{x}{\alpha}, \frac{y}{\alpha}, \frac{z}{\alpha} ; t ; k, \beta\right)\right]^{\alpha}=f_{a, b}^{(\alpha)}(x, y, z ; t ; k, \beta) .
$$

Using (4.1), we start by proving the following closed form summation formula:

Theorem 4.1 Let the conditions (i)-(iii) be satisfied. The following summation formula:

$$
\begin{gathered}
\sum_{l=0}^{n}\left(\begin{array}{c}
n \\
l
\end{array}\right)\left[{ }_{H} P_{n-l+1, \beta}^{(\alpha)}(x, y, z ; k, a, b)_{H} P_{l, \beta}\left(\frac{x}{\alpha}, \frac{y}{\alpha}, \frac{z}{\alpha} ; k, a, b\right)\right. \\
\left.-\alpha_{H} P_{n-l, \beta}^{(\alpha)}(x, y, z ; k, a, b)_{H} P_{l+1, \beta}\left(\frac{x}{\alpha}, \frac{y}{\alpha}, \frac{z}{\alpha} ; k, a, b\right)\right]=0
\end{gathered}
$$

holds true.

Proof Taking logarithms on both sides of (4.1) and then differentiating with respect to $t$, we get

$$
\begin{aligned}
& \frac{\partial f_{a, b}^{(\alpha)}(x, y, z ; t ; k, \beta)}{\partial t} f_{a, b}^{(1)}\left(\frac{x}{\alpha}, \frac{y}{\alpha}, \frac{z}{\alpha} ; t ; k, \beta\right) \\
& =\alpha f_{a, b}^{(\alpha)}(x, y, z ; t ; k, \beta) \frac{\partial f_{a, b}^{(1)}\left(\frac{x}{\alpha}, \frac{y}{\alpha}, \frac{z}{\alpha} ; t ; k, \beta\right)}{\partial t} .
\end{aligned}
$$

Inserting the corresponding generating relations, we obtain

$$
\begin{aligned}
& \sum_{n=1}^{\infty} n_{H} P_{n, \beta}^{(\alpha)}(x, y, z ; k, a, b) \frac{t^{n-1}}{n !} \sum_{l=0}^{\infty}{ }_{H} P_{l, \beta}\left(\frac{x}{\alpha}, \frac{y}{\alpha}, \frac{z}{\alpha} ; k, a, b\right) \frac{t^{l}}{l !} \\
& \quad=\alpha \sum_{n=0}^{\infty}{ }_{H} P_{n, \beta}^{(\alpha)}(x, y, z ; k, a, b) \frac{t^{n}}{n !} \sum_{l=0}^{\infty} l_{H} P_{l, \beta}\left(\frac{x}{\alpha}, \frac{y}{\alpha}, \frac{z}{\alpha} ; k, a, b\right) \frac{t^{l-1}}{l !}
\end{aligned}
$$

and hence

$$
\begin{aligned}
& \sum_{n=0}^{\infty}{ }_{H} P_{n+1, \beta}^{(\alpha)}(x, y, z ; k, a, b) \frac{t^{n}}{n !} \sum_{l=0}^{\infty}{ }_{H} P_{l, \beta}\left(\frac{x}{\alpha}, \frac{y}{\alpha}, \frac{z}{\alpha} ; k, a, b\right) \frac{t^{l}}{l !} \\
& =\alpha \sum_{n=0}^{\infty}{ }_{H} P_{n, \beta}^{(\alpha)}(x, y, z ; k, a, b) \frac{t^{n}}{n !} \sum_{l=0}^{\infty}{ }_{H} P_{l+1, \beta}\left(\frac{x}{\alpha}, \frac{y}{\alpha}, \frac{z}{\alpha} ; k, a, b\right) \frac{t^{l}}{l !} .
\end{aligned}
$$


Using the fact that (see [34, p.101, Lemma 3])

$$
\sum_{n=0}^{\infty} \sum_{l=0}^{\infty} A(n, l)=\sum_{n=0}^{\infty} \sum_{l=0}^{n} A(n-l, l)
$$

we get

$$
\begin{aligned}
& \sum_{n=0}^{\infty}\left[\sum_{l=0}^{n}\left(\begin{array}{l}
n \\
l
\end{array}\right)_{H} P_{n-l+1, \beta}^{(\alpha)}(x, y, z ; k, a, b)_{H} P_{l, \beta}\left(\frac{x}{\alpha}, \frac{y}{\alpha}, \frac{z}{\alpha} ; k, a, b\right)\right] \frac{t^{n}}{n !} \\
& \quad=\alpha \sum_{n=0}^{\infty}\left[\sum_{l=0}^{n}\left(\begin{array}{l}
n \\
l
\end{array}\right){ }_{H} P_{n-l, \beta}^{(\alpha)}(x, y, z ; k, a, b)_{H} P_{l+1, \beta}\left(\frac{x}{\alpha}, \frac{y}{\alpha}, \frac{z}{\alpha} ; k, a, b\right)\right] \frac{t^{n}}{n !}
\end{aligned}
$$

Whence the result.

Corollary 4.2 Let $k=a=b=1$ and $\beta=\lambda$. For all $m \in \mathbb{N}, n \in \mathbb{N}_{0}, \lambda \in \mathbb{C}$, we have the following closed form summation formula for the generalized Apostol-Bernoulli polynomials:

$$
\begin{gathered}
\sum_{k=0}^{n}\left(\begin{array}{l}
n \\
k
\end{array}\right)\left[{ }_{H} \mathcal{B}_{n-k+1}^{(\alpha)}(x, y, z ; \lambda)_{H} \mathcal{B}_{k}\left(\frac{x}{\alpha}, \frac{y}{\alpha}, \frac{z}{\alpha} ; \lambda\right)\right. \\
\left.-\alpha_{H} \mathcal{B}_{n-k}^{(\alpha)}(x, y, z ; \lambda) \mathcal{B}_{k+1}\left(\frac{x}{\alpha}, \frac{y}{\alpha}, \frac{z}{\alpha} ; \lambda\right)\right]=0 .
\end{gathered}
$$

Corollary 4.3 Let $k+1=-a=b=1$ and $\beta=\lambda$. For all $m \in \mathbb{N}, n \in \mathbb{N}_{0}, \lambda \in \mathbb{C}$, we have the following closed form summation formula for the generalized Apostol-Euler polynomials:

$$
\begin{gathered}
\sum_{k=0}^{n}\left(\begin{array}{l}
n \\
k
\end{array}\right)\left[{ }_{H} \mathcal{E}_{n-k+1}^{(\alpha)}(x, y, z ; \lambda)_{H} \mathcal{E}_{k}\left(\frac{x}{\alpha}, \frac{y}{\alpha}, \frac{z}{\alpha} ; \lambda\right)\right. \\
\left.-\alpha_{H} \mathcal{E}_{n-k}^{(\alpha)}(x, y, z ; \lambda) \mathcal{E}_{k+1}\left(\frac{x}{\alpha}, \frac{y}{\alpha}, \frac{z}{\alpha} ; \lambda\right)\right]=0 .
\end{gathered}
$$

Corollary 4.4 Let $k=-2 a=b=1$ and $2 \beta=\lambda$. For all $m \in \mathbb{N}, n \in \mathbb{N}_{0}, \lambda \in \mathbb{C}$, we have the following closed form summation formula for the generalized Apostol-Genocchi polynomials:

$$
\begin{array}{r}
\sum_{k=0}^{n}\left(\begin{array}{l}
n \\
k
\end{array}\right)\left[{ }_{H} \mathcal{G}_{n-k+1}^{(\alpha)}(x, y, z ; \lambda)_{H} \mathcal{G}_{k}\left(\frac{x}{\alpha}, \frac{y}{\alpha}, \frac{z}{\alpha} ; \lambda\right)\right. \\
\left.-\alpha_{H} \mathcal{G}_{n-k}^{(\alpha)}(x, y, z ; \lambda) \mathcal{G}_{k+1}\left(\frac{x}{\alpha}, \frac{y}{\alpha}, \frac{z}{\alpha} ; \lambda\right)\right]=0 .
\end{array}
$$

Theorem 4.5 Let the conditions (i)-(iii) be satisfied. Then we have the following relation between Hermite based Apostol polynomials and 3d-Hermite polynomials:

$$
\begin{aligned}
{ }_{H} P_{n+m, \beta}^{(\alpha)}(X, Y, Z ; k, a, b) \\
\quad=\sum_{r, l=0}^{n, m}\left(\begin{array}{c}
n \\
r
\end{array}\right)\left(\begin{array}{c}
m \\
l
\end{array}\right) H_{r+l}^{(3)}(X-x, Y-y, Z-z)_{H} P_{n+m-r-l}^{(\alpha)}(x, y, z ; k, a, b) .
\end{aligned}
$$


Proof From (2.1), we can write that

$$
\begin{aligned}
\left(\frac{2^{1-k}(t+w)^{k}}{\beta^{b} e^{t+w}-a^{b}}\right)^{\alpha} e^{x(t+w)+y(t+w)^{2}+z(t+w)^{3}} & =\sum_{n=0}^{\infty}{ }_{H} P_{n, \beta}^{(\alpha)}(x, y, z ; k, a, b) \frac{(t+w)^{n}}{n !} \\
& =\sum_{n, m=0}^{\infty}{ }_{H} P_{n+m, \beta}^{(\alpha)}(x, y, z ; k, a, b) \frac{t^{n}}{n !} \frac{w^{m}}{m !}
\end{aligned}
$$

Therefore, we get

$$
\left(\frac{2^{1-k}(t+w)^{k}}{\beta^{b} e^{t+w}-a^{b}}\right)^{\alpha}=e^{-x(t+w)-y(t+w)^{2}-z(t+w)^{3}} \sum_{n, m=0}^{\infty}{ }_{H} P_{n+m, \beta}^{(\alpha)}(x, y, z ; k, a, b) \frac{t^{n}}{n !} \frac{w^{m}}{m !}
$$

Multiplying both sides by $e^{X(t+w)+Y(t+w)^{2}+Z(t+w)^{3}}$, we have

$$
\begin{aligned}
& \left(\frac{2^{1-k}(t+w)^{k}}{\beta^{b} e^{t+w}-a^{b}}\right)^{\alpha} e^{X(t+w)+Y(t+w)^{2}+Z(t+w)^{3}} \\
& \quad=e^{(X-x)(t+w)+(Y-y)(t+w)^{2}+(Z-z)(t+w)^{3}} \sum_{n, m=0}^{\infty}{ }_{H} P_{n+m, \beta}^{(\alpha)}(x, y, z ; k, a, b) \frac{t^{n}}{n !} \frac{w^{m}}{m !} .
\end{aligned}
$$

Taking into account (1.1) and (4.3), then using (4.2), we get

$$
\begin{aligned}
& \sum_{n, m=0}^{\infty}{ }_{H} P_{n+m, \beta}^{(\alpha)}(X, Y, Z ; k, a, b) \frac{t^{n}}{n !} \frac{w^{m}}{m !} \\
& \quad=\sum_{n, m=0}^{\infty} H_{n}^{(\alpha)} P_{n+m, \beta}(x, y, z ; k, a, b) \frac{t^{n}}{n !} \frac{w^{m}}{m !} \sum_{r, l=0}^{\infty} H_{r+l}^{(3)}(X-x, Y-y, Z-z) \frac{t^{r}}{r !} \frac{w^{l}}{l !} \\
& =\sum_{n, m=0}^{\infty} \sum_{r, l=0}^{n, m}\left(\begin{array}{c}
n \\
r
\end{array}\right)\left(\begin{array}{c}
m \\
l
\end{array}\right) H_{r+l}^{(3)}(X-x, Y-y, Z-z)_{H} P_{n+m-r-l}^{(\alpha)}(x, y, z ; k, a, b) \frac{t^{n}}{n !} \frac{w^{m}}{m !}
\end{aligned}
$$

Whence the result.

Corollary 4.6 Let $k=a=b=1$ and $\beta=\lambda$. For all $c, d, m \in \mathbb{N}, n \in \mathbb{N}_{0}, \lambda \in \mathbb{C}$, we have the following summation formula between the Hermite-based generalized Apostol-Bernoulli polynomials and 3d-Hermite polynomials:

$$
\begin{aligned}
{ }_{H} \mathcal{B}_{n+m}^{(\alpha)}(X, Y, Z ; \lambda) \\
\quad=\sum_{k, l=0}^{n, m}\left(\begin{array}{l}
n \\
k
\end{array}\right)\left(\begin{array}{c}
m \\
l
\end{array}\right) H_{k+l}^{(3)}(X-x, Y-y, Z-z)_{H} \mathcal{B}_{n+m-k-l}^{(\alpha)}(x, y, z ; \lambda) .
\end{aligned}
$$

Corollary 4.7 Let $k+1=-a=b=1$ and $\beta=\lambda$. For all $m \in \mathbb{N}, n \in \mathbb{N}_{0}, \lambda \in \mathbb{C}$, we have the following summation formula between the Hermite-based generalized Apostol-Euler polynomials and 3d-Hermite polynomials:

$$
\begin{aligned}
{ }_{H} \mathcal{E}_{n+m}^{(\alpha)}(X, Y, Z ; \lambda) \\
\quad=\sum_{k, l=0}^{n, m}\left(\begin{array}{l}
n \\
k
\end{array}\right)\left(\begin{array}{c}
m \\
l
\end{array}\right) H_{k+l}^{(3)}(X-x, Y-y, Z-z)_{H} \mathcal{E}_{n+m-k-l}^{(\alpha)}(x, y, z ; \lambda) .
\end{aligned}
$$


Corollary 4.8 Let $k=-2 a=b=1$ and $2 \beta=\lambda$. For all $m \in \mathbb{N}, n \in \mathbb{N}_{0}, \lambda \in \mathbb{C}$, we have the following summation formula between the Hermite-based generalized Apostol-Genocchi polynomials and 3d-Hermite polynomials:

$$
\begin{aligned}
& { }_{H} \mathcal{G}_{n+m}^{(\alpha)}(X, Y, Z ; \lambda) \\
& \quad=\sum_{k, l=0}^{n, m}\left(\begin{array}{l}
n \\
k
\end{array}\right)\left(\begin{array}{c}
m \\
l
\end{array}\right) H_{k+l}^{(3)}(X-x, Y-y, Z-z)_{H} \mathcal{G}_{n+m-k-l}^{(\alpha)}(x, y, z ; \lambda) .
\end{aligned}
$$

\section{Competing interests}

The author declares that they have no competing interests.

\section{Author's contributions}

The author completed the paper himself. The author read and approved the final manuscript.

\section{Acknowledgements}

Dedicated to Professor Hari M Srivastava.

\section{Received: 3 December 2012 Accepted: 10 April 2013 Published: 24 April 2013}

\section{References}

1. Khan, S, Yasmin, G, Khan, R, Hassan, NAM: Hermite-based Appell polynomials: properties and applications. J. Math. Anal. Appl. 351, 756-764 (2009)

2. Özarslan, MA: Unified Apostol-Bernoulli, Euler and Genocchi polynomials. Comput. Math. Appl. 62(6), $2452-2462$ (2011)

3. Ozden, $H$, Simsek, Y, Srivastava, HM: A unified presentation of the generating functions of the generalized Bernoulli, Euler and Genocchi polynomials. Comput. Math. Appl. 60(10), 2779-2787 (2010)

4. Luo, Q-M: On the Apostol-Bernoulli polynomials. Cent. Eur. J. Math. 2(4), 509-515 (2004)

5. Luo, Q-M, Srivastava, HM: Some relationships between the Apostol-Bernoulli and Apostol-Euler polynomials. Comput. Math. Appl. 51(3-4), 631-642 (2006)

6. Luo, Q-M, Srivastava, HM: Some generalizations of the Apostol-Bernoulli and Apostol-Euler polynomials. J. Math. Anal. Appl. 308(1), 290-302 (2005)

7. Srivastava, HM: Some formulas for the Bernoulli and Euler polynomials at rational arguments. Math. Proc. Camb. Philos. Soc. 129(1), 77-84 (2000)

8. Luo, Q-M: Apostol-Euler polynomials of higher order and Gaussian hypergeometric functions. Taiwan. J. Math. 10 917-925 (2006)

9. Luo, Q-M: Extension for the Genocchi polynomials and its Fourier expansions and integral representations. Osaka J. Math. 48(2), 291-309 (2011)

10. Luo, Q-M: Fourier expansions and integral representations for the Genocchi polynomials. J. Integer Seq. 12, Article ID 09.1.4 (2009)

11. Dere, R, Simsek, Y: Genocchi polynomials associated with the umbral algebra. Appl. Math. Comput. 218, 756-761 (2011)

12. Dere, R, Simsek, Y: Applications of umbral algebra to some special polynomials. Adv. Stud. Contemp. Math. 22, 433-438 (2012)

13. Karande, BK, Thakare, NK: On the unification of Bernoulli and Euler polynomials. Indian J. Pure Appl. Math. 6, 98-107 (1975)

14. Kurt, B, Simsek, Y: Frobenius-Euler type polynomials related to Hermite-Bernoulli polynomials. Analysis and applied math. AIP Conf. Proc. 1389, 385-388 (2011)

15. Luo, Q-M: q-Extensions for the Apostol-Genocchi polynomials. Gen. Math. 17, 113-125 (2009)

16. Luo, Q-M: The multiplication formulas for the Apostol-Bernoulli and Apostol-Euler polynomials of higher order. Integral Transforms Spec. Funct. 20(5-6), 377-391 (2009)

17. Luo, Q-M, Srivastava, HM: Some generalizations of the Apostol-Genocchi polynomials and the Stirling numbers of the second kind. Appl. Math. Comput. 217, 5702-5728 (2011)

18. Ozden, $H$, Simsek, Y: A new extension of $q$-Euler numbers and polynomials related to their interpolation functions. Appl. Math. Lett. 21, 934-939 (2008)

19. Simsek, Y: Complete sum of products of $(h, q)$-extension of Euler polynomials and numbers. J. Differ. Equ. Appl. 16(11), 1331-1348 (2010)

20. Simsek, Y: Twisted $(h, q)$-Bernoulli numbers and polynomials related to twisted $(h, q)$-zeta function and $L$-function J. Math. Anal. Appl. 324, 790-804 (2006)

21. Simsek, Y: Twisted $p$-adic (h, q)-L-functions. Comput. Math. Appl. 59, 2097-2110 (2010)

22. Srivastava, HM: Some generalizations and basic (or q-) extensions of the Bernoulli, Euler and Genocchi polynomials. Appl. Math. Inf. Sci. 5, 390-444 (2011)

23. Srivastava, HM, Choi, J: Series Associated with the Zeta and Related Functions. Kluwer Academic, Dordrecht (2001)

24. Srivastava, HM, Choi, J: Zeta and q-Zeta Functions and Associated Series and Integrals. Elsevier, Amsterdam (2012)

25. Srivastava, HM, Garg, M, Choudhary, S: A new generalization of the Bernoulli and related polynomials. Russ. J. Math. Phys. 17, 251-261 (2010) 
26. Srivastava, HM, Özarslan, MA, Kaanoğlu, C: Some generalized Lagrange-based Apostol-Bernoulli, Apostol-Euler and Apostol-Genocchi polynomials. Russ. J. Math. Phys. 20, 110-120 (2013)

27. Zhang, Z, Yang, H: Several identities for the generalized Apostol-Bernoulli polynomials. Comput. Math. Appl. 56(12), 2993-2999 (2008)

28. Deeba, E, Rodriguez, D: Stirling's series and Bernoulli numbers. Am. Math. Mon. 98, 423-426 (1991)

29. Kurt, V: A further symmetric relation on the analogue of the Apostol-Bernoulli and the analogue of the Apostol-Genocchi polynomials. Appl. Math. Sci. 3(53-56), 2757-2764 (2009)

30. Raabe, JL: Zurückführung einiger Summen und bestimmten Integrale auf die Jakob Bernoullische Function. J. Reine Angew. Math. 42, 348-376 (1851)

31. Tuenter, HJH: A symmetry of power sum polynomials and Bernoulli numbers. Am. Math. Mon. 108, $258-261$ (2001)

32. Yang, SL: An identity of symmetry for the Bernoulli polynomials. Discrete Math. 308(4), 550-554 (2008)

33. Srivastava, HM, Özarslan, MA, Kaanoğlu, C: Some families of generating functions for a certain class of three-variable polynomials. Integral Transforms Spec. Funct. 21(12), 885-896 (2010)

34. Srivastava, HM, Manocha, HL: A Treatise on Generating Functions. Halsted, New York (1984)

doi:10.1186/1687-1847-2013-116

Cite this article as: Özarslan: Hermite-based unified Apostol-Bernoulli, Euler and Genocchi polynomials. Advances in Difference Equations 2013 2013:116.

\section{Submit your manuscript to a SpringerOpen ${ }^{\circ}$ journal and benefit from:}

- Convenient online submission

Rigorous peer review

- Immediate publication on acceptance

- Open access: articles freely available online

- High visibility within the field

- Retaining the copyright to your article 